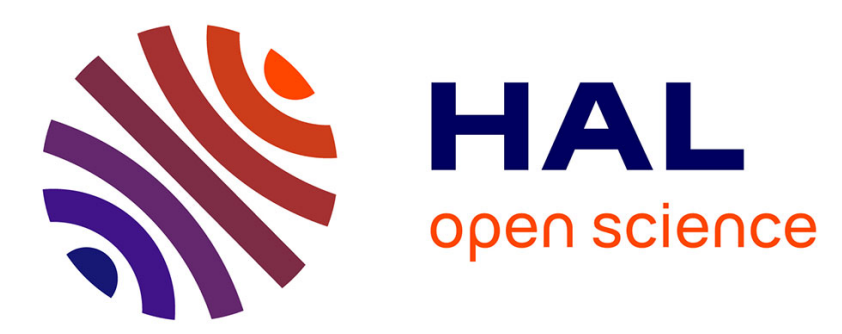

\title{
A method to extract pure Raman spectrum of epitaxial graphene on $\mathrm{SiC}$
}

Jan Kunc, Yike Hu, James Palmer, Claire Berger, Walter A. de Heer

\section{To cite this version:}

Jan Kunc, Yike Hu, James Palmer, Claire Berger, Walter A. de Heer. A method to extract pure Raman spectrum of epitaxial graphene on SiC. Applied Physics Letters, 2013, 103 (20), pp.201911. 10.1063/1.4830374 . hal-00911210

\section{HAL Id: hal-00911210 https://hal.science/hal-00911210}

Submitted on 26 May 2021

HAL is a multi-disciplinary open access archive for the deposit and dissemination of scientific research documents, whether they are published or not. The documents may come from teaching and research institutions in France or abroad, or from public or private research centers.
L'archive ouverte pluridisciplinaire HAL, est destinée au dépôt et à la diffusion de documents scientifiques de niveau recherche, publiés ou non, émanant des établissements d'enseignement et de recherche français ou étrangers, des laboratoires publics ou privés. 


\section{A method to extract pure Raman spectrum of epitaxial graphene on SiC}

Cite as: Appl. Phys. Lett. 103, 201911 (2013); https://doi.org/10.1063/1.4830374

Submitted: 26 June 2013 . Accepted: 30 October 2013 . Published Online: 15 November 2013

J. Kunc, Y. Hu, J. Palmer, C. Berger, and W. A. de Heer
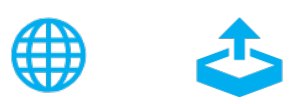

View Online

Export Citation

\section{ARTICLES YOU MAY BE INTERESTED IN}

Raman spectra of epitaxial graphene on $\mathrm{SiC}(0001)$

Applied Physics Letters 92, 201918 (2008); https://doi.org/10.1063/1.2929746

The quasi-free-standing nature of graphene on $\mathrm{H}$-saturated $\mathrm{SiC}(0001)$

Applied Physics Letters 99, 122106 (2011); https://doi.org/10.1063/1.3643034

Hydrogen intercalation of epitaxial graphene and buffer layer probed by mid-infrared absorption and Raman spectroscopy

AIP Advances 8, 045015 (2018); https://doi.org/10.1063/1.5024132

\section{Challenge us.}

What are your needs for periodic signal detection?

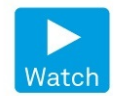

- Zurich

Instruments

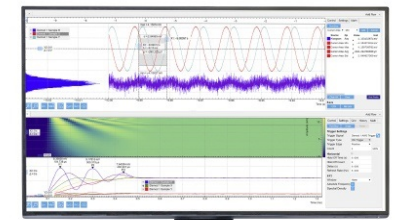

(0 


\title{
A method to extract pure Raman spectrum of epitaxial graphene on SiC
}

\author{
J. Kunc, ${ }^{1,2, a)}$ Y. Hu, ${ }^{1}$ J. Palmer, ${ }^{1}$ C. Berger, ${ }^{1,3}$ and W. A. de Heer ${ }^{1}$ \\ ${ }^{1}$ The Georgia Institute of Technology, Atlanta, Georgia 30332-0430, USA \\ ${ }^{2}$ Faculty of Mathematics and Physics, Institute of Physics, 12116 Prague, Czech Republic \\ ${ }^{3}$ CNRS/Institute Néel, BP 166, 38042 Grenoble, France
}

(Received 26 June 2013; accepted 30 October 2013; published online 15 November 2013)

\begin{abstract}
The Raman spectrum of epitaxial graphene on $\mathrm{SiC}$ is generally obtained by simply subtracting a $\mathrm{SiC}$ spectra from the experimental data, which results in noisy spectrum and negative intensity. By using a Non-negative Matrix Factorization (NMF) method, we obtain pure graphene spectra, even for monolayer graphene and sub-micron size patterned features, as well as in spatial mapping and depth profile. We show that the NMF method is efficient in data smoothing and for signal deconvolution with no assumption required for the functional form of the signals. C 2013 AIP Publishing LLC. [http://dx.doi.org/10.1063/1.4830374]
\end{abstract}

As graphene is getting more attention as an electronic material, ${ }^{1}$ so is the need for a better sample characterization. Epitaxial graphene (EG) grown on $4 \mathrm{H}-$ or $6 \mathrm{H}-\mathrm{SiC}$ can be produced with a very high structural quality on mono crystalline semiconductor wafer allowing for large scale integration. ${ }^{2,3}$ Excellent electronic properties have been demonstrated in epitaxial graphene, which most recently record high frequency transistor operation ${ }^{4,5}$ and spin diffusion lengths for spintronics. ${ }^{6}$ Smoothed-edge nanoscale ribbons ${ }^{2,7}$ can also be produced on non-polar $\mathrm{SiC}$ facets that present exceptional conducting properties ${ }^{7,8}$ and wide band-gap semiconducting strips. ${ }^{9}$

A widely used primary characterization of graphene is Raman spectroscopy. ${ }^{10,11}$ However, unlike graphene transferred to $\mathrm{SiO}_{2}$, the Raman spectra of EG on $\mathrm{SiC}$ consist of a combination of signals from graphene and bulk $\mathrm{SiC}$ in the spectral region of the graphene D- and G-peaks. ${ }^{12-16}$ This significantly complicates the data interpretation. The Raman spectra of $\mathrm{SiC}$ and graphene are usually separated by a simple subtraction of a SiC bulk spectrum. ${ }^{17,18}$ The signal resulting from subtraction is however poorly defined ${ }^{17,18}$ and is often noisy, showing nonphysical negative intensity, specially when the SiC and graphene peaks are of similar intensity, that is for very thin graphene films.

Another issue is related to the Raman spectroscopy of patterned $\mu \mathrm{m}$-scale electronic devices. Unless in the nearfield regime, Raman scattering mapping is limited by the spatial resolution given by the laser beam size (at best about $\mu \mathrm{m}$ spot). Spatial resolution can, in principle, be increased to the sub- $\mu \mathrm{m}$ scale by data deconvolution, but the laser beam profile (impulse response function) has to be known.

In this Letter, we demonstrate that the Raman spectra of epitaxial graphene can be decomposed very well into pure graphene and SiC spectra by using a recently developed Nonnegative Matrix Factorization ${ }^{19}$ (NMF) method (software freely accessible $\left.{ }^{20,21}\right)$. The graphene and SiC Raman spectra can be identified as the NMF components, and the resulting graphene spectra are clean, well-resolved and smooth, even in the spectral range where $\mathrm{SiC}$ and graphene peaks overlap. We also apply the method to a patterned sample where

a)jan.kunc@physics.gatech.edu multilayers pads connect to single layer graphene and show that spatial maps of the NMF spectral components correlate with that of local electrostatic force microscopy (EFM) images. We also show that the method can be used for data smoothing. Finally, we demonstrate that NMF is a good alternative to signal deconvolution because the method does not require a priori knowledge of an impulse response function. As a further application, we propose an experimental technique to characterize the spatial spread of the laser beams.

We have used the NMF method to analyze Raman data for 15 epitaxial graphene samples grown on $\mathrm{SiC}$ by the confinement controlled sublimation method. ${ }^{22}$ Here, we focus on two representative samples, grown on the $4 \mathrm{H}-\mathrm{SiC}(000 \overline{1})$ carbon-face. The first sample is a C-face multilayer epitaxial graphene $(\mathrm{MEG})$ sample of $\approx 5$ layers. For the second sample, MEG was plasma etched away everywhere except in two $10 \times 10 \mu \mathrm{m}^{2} \mathrm{MEG}$ areas separated by a $0.9 \mu \mathrm{m}$ wide channel. A sub-single layer graphene was subsequently grown in the channel and around the MEG areas. This provides three regions on the same sample: $\approx 10$ layer thick MEG pads, single graphene layer, and bare $\mathrm{SiC}$ areas.

Raman scattering was excited by a $\lambda=532 \mathrm{~nm}$ laser light with laser beam size of $\approx 1 \mu \mathrm{m}^{2}$ (Horiba Jobin-Yvon LabRam). The data were taken between $1000 \mathrm{~cm}^{-1}$ and $3600 \mathrm{~cm}^{-1}$ with a spectral resolution of $1 \mathrm{~cm}^{-1}$. We present below 3 sets of experiments.

In the first experiment (Fig. 1), we demonstrate the decomposition of the Raman spectra from the first MEG sample onto a $\mathrm{SiC}$ and a graphene spectrum. For this $m=69$, Raman spectra were taken, each at a different focal plane of the laser beam, between $z=10 \mu \mathrm{m}$ above the surface down to $z=-25 \mu \mathrm{m}$ below, in steps of $\Delta z=0.5$ to $1 \mu \mathrm{m}$. The focal plane can be adjusted with respect to the sample surface with a precision of $\pm 0.25 \mu \mathrm{m}$. This allows to change the relative intensity of the graphene and $\mathrm{SiC}$ signals. The exact $z$ position is not important as long as a sufficient number of spectra are taken at the graphene plane and deep below it (i.e., where the graphene or $\mathrm{SiC}$ signal prevails, respectively).

For the second sample, we identify three regions of various graphene thicknesses. In this case, we acquired $m=828$ spectra by mapping the surface at constant $z=0 \mu \mathrm{m}$. The surface area $3.4 \times 9 \mu \mathrm{m}^{2}$ was scanned with a 


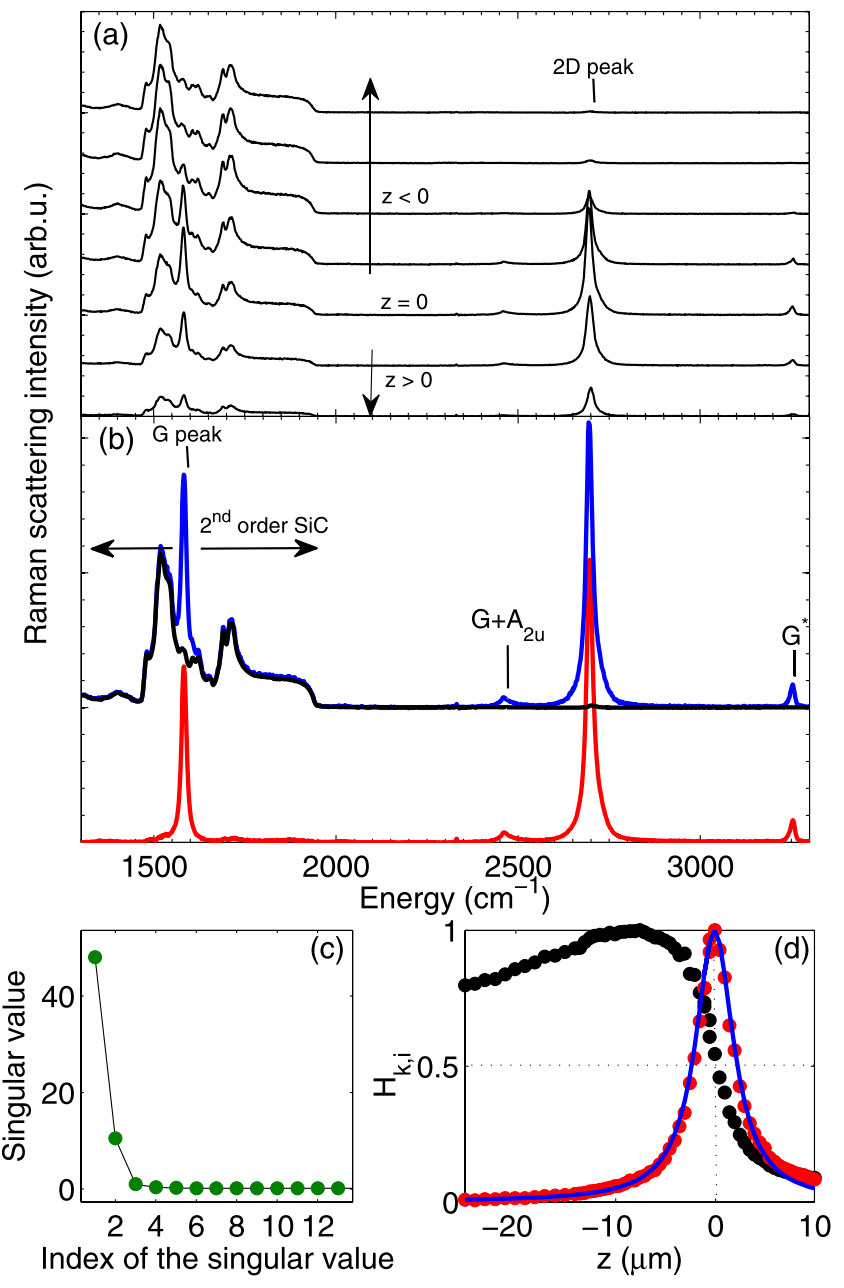

FIG. 1. (a) Representative 7 out of 69 Raman spectra used for the NMF decomposition. The focal plane was changed from $z=10 \mu \mathrm{m}$ above $(z>0)$ down to $z=-25 \mu \mathrm{m}$ below $(z<0)$ the sample surface. (b) Black (SiC) and red (graphene) decomposed spectra are compared with blue Raman spectrum at $z=0 \mu \mathrm{m}$ before decomposition. (c) First largest singular values of 69 Raman spectra used for NMF decomposition. (d) Linear coefficients (relative strengths) of graphene (red) and $\mathrm{SiC}$ (black) basis functions versus focal plane distance from sample surface. The blue curve is a fit assuming Gaussian beam of the excitation laser (Rayleigh parameter $z_{R}=2.3 \mu \mathrm{m}$ ).

lateral resolution $0.2 \mu \mathrm{m}$ (18 and 46 steps in $x$ and $y$ lateral direction, respectively).

In short, the NMF is a multivariate analysis tool providing data categorization as $\mathrm{K}$-means ${ }^{23}$ and spectral decomposition on a basis of eigenvectors (decomposition known as Principal Component Analysis (PCA)). In contrast to PCA, which decomposes data into an orthonormal basis, NMF imposes non-negativity constraint, thus facilitating the interpretation of the basis functions. (The Raman spectral intensity cannot be physically negative.) We use freely accessible implementation of $\mathrm{NMF}^{20,21}$ using both Block Principal Pivoting and Active $\mathrm{Set}^{21}$ minimization algorithms. Beside non-negativity constraints, constraints on sparsity ${ }^{24}$ and regularity ${ }^{20}$ of the basis functions and/or linear coefficients have been applied.

In both experiments, all the spectra were normalized by their global maximum (maximal Raman scattering intensity within all 69 or 828 spectra, respectively), and a local constant background was subtracted from each spectrum separately. The data were organized as columns of matrix $V_{\text {exp }}$.
The matrices $V_{\text {exp }}$ were decomposed by NMF giving elementwise non-negative matrices $H$ and $W$ such that $V_{\text {exp }}=W H+E$. The error matrix $E$, which Frobenius norm is minimized by the NMF algorithm, contains the noise information and the matrix $V=W H$ the smoothed experimental data. Data smoothing by NMF is discussed at the end of this Letter (Fig. 3). The columns of matrix $W$ are the basis functions (the EG and SiC spectra for instance) and rows of matrix $H$ are the corresponding linear coefficients ascribing the weight of the basis function in each experimental spectrum. The matrices $\mathrm{W}$ and $\mathrm{H}$ are therefore of rank $n \times k$ and $k \times m$, respectively, where $k$ is the number of basis functions, $n$ is the number of data points in each experimental spectrum, and $m$ is the number of experimental spectra. The number of basis functions $k$ is determined by the number of largest singular values of the matrix $V_{\text {exp }}$ factorized by Singular Value Decomposition (SVD).

A few representative Raman spectra as a function of the relative position $z$ for the first MEG sample are shown in Fig. 1(a). Each spectrum has a different $\mathrm{SiC}$ relative to graphene weight. When the excitation beam is focused at the sample surface ( $z=0 \mu \mathrm{m}$ in Fig. 1(a) and blue curve in Fig. 1(b)), the Raman spectrum consists of a combination of 2 nd order bulk SiC Refs. 25 and 26) and $\mathrm{G}, \mathrm{G}+\mathrm{A}_{2 u}, 2 \mathrm{D}$, and $\mathrm{G}^{*}$ graphene Raman peaks. ${ }^{11,27}$ The missing graphene D-peak indicates good quality graphene. Note the single 2D graphene peak indicating that the 5 layers in the MEG sample are not stacked like in graphite, as previously discussed. ${ }^{12,17}$ As expected, the overall Raman signal intensity is weak when the focal plane is high above the sample $(z>0)$ and increases when approaching the surface $(z \rightarrow 0 \mu \mathrm{m})$. Lowering the focal plane below surface leads to attenuation (enhancement) of the graphene ( $\mathrm{SiC}$, respectively) signal. Far below the surface $(z<-10 \mu \mathrm{m})$, the $\mathrm{SiC}$ signal intensity slightly reduces due to light absorption in the $\mathrm{SiC}$ substrate.

The first 13 principal values of matrix $V_{\text {exp }}$ obtained by SVD are plotted in Fig. 1(c). The two largest principal values represent the two principal components, as seen in Fig. 1(c), the others have negligible contribution. The matrix $V_{\text {exp }}$ is then factorized with NMF of rank $k=2$, giving two nonnegative basis functions (columns of matrix $W$ ) plotted in red and black in Fig. 1(b). The basis functions are attributed to the $\mathrm{SiC}$ and graphene Raman spectra. The linear coefficients (the 2 rows of matrix $H$, plotted in Fig. 1(d)) show the relative contributions of $\mathrm{SiC}$ (black dots) and graphene (red dots) to the measured Raman spectra (Fig. 1(a)) as a function of $z$. As expected, the graphene signal is maximized for $z=0$, i.e., when the excitation laser beam is focused at the sample surface.

Assuming a Gaussian shape for the laser beam, ${ }^{28}$ the $z$-dependence of the graphene signal can be fitted by $H_{1, i}=\frac{1}{1+\left(z / z_{R}\right)^{2}}$. Here, $H_{1, i}$ is the row of linear coefficients for graphene as determined by NMF, $z_{R}=\frac{\pi w_{0}^{2}}{\lambda}=2.3 \mu \mathrm{m}$ is a Rayleigh parameter of the Gaussian beam, and $2 w_{0}=1.2 \mu \mathrm{m}$ is the beam waist (i.e., the radial width). The beam Full Width at Half Maximum FWHM $=1.2 \mu \mathrm{m}$ confirms the expected beam waist $\approx 1 \mu \mathrm{m}$ (Jobin Yvon specification).

Note that for performing spectral decomposition only, the number of spectra (here $m=69$ ) can be greatly reduced, as long as the condition $(n+m) k<n m$ is fulfilled ${ }^{19}$ 
(here, $n=1867$ data points per spectrum). The large $m$ was only necessary here to get enough spatial resolution to characterize the laser beam.

We now show how NMF can be used as an alternative to deconvolution. The second (patterned) sample was mapped with Atomic Force Microscopy (AFM), EFM (Fig. 2(a)), and Raman scattering. Three different graphene/SiC areas are identified in the EFM image (Fig. 2(a)). The brown areas in Fig. 2(a) (area 3) are the MEG pads, the light areas (such as 1) are single layer graphene, and the dark one (labelled 2) is non-graphitized $\mathrm{SiC}$ substrate. The normalized Raman spectra measured at points 1,2 , and 3 are plotted as dashed lines in Fig. 2(e). The D, G, and 2D graphene peaks are clearly identified in the spectra, as well as the $\mathrm{SiC}$ Raman peaks. The $2 \mathrm{D}$ peak in area 3 presents a double peak structure which is sometimes observed in MEG sample. It
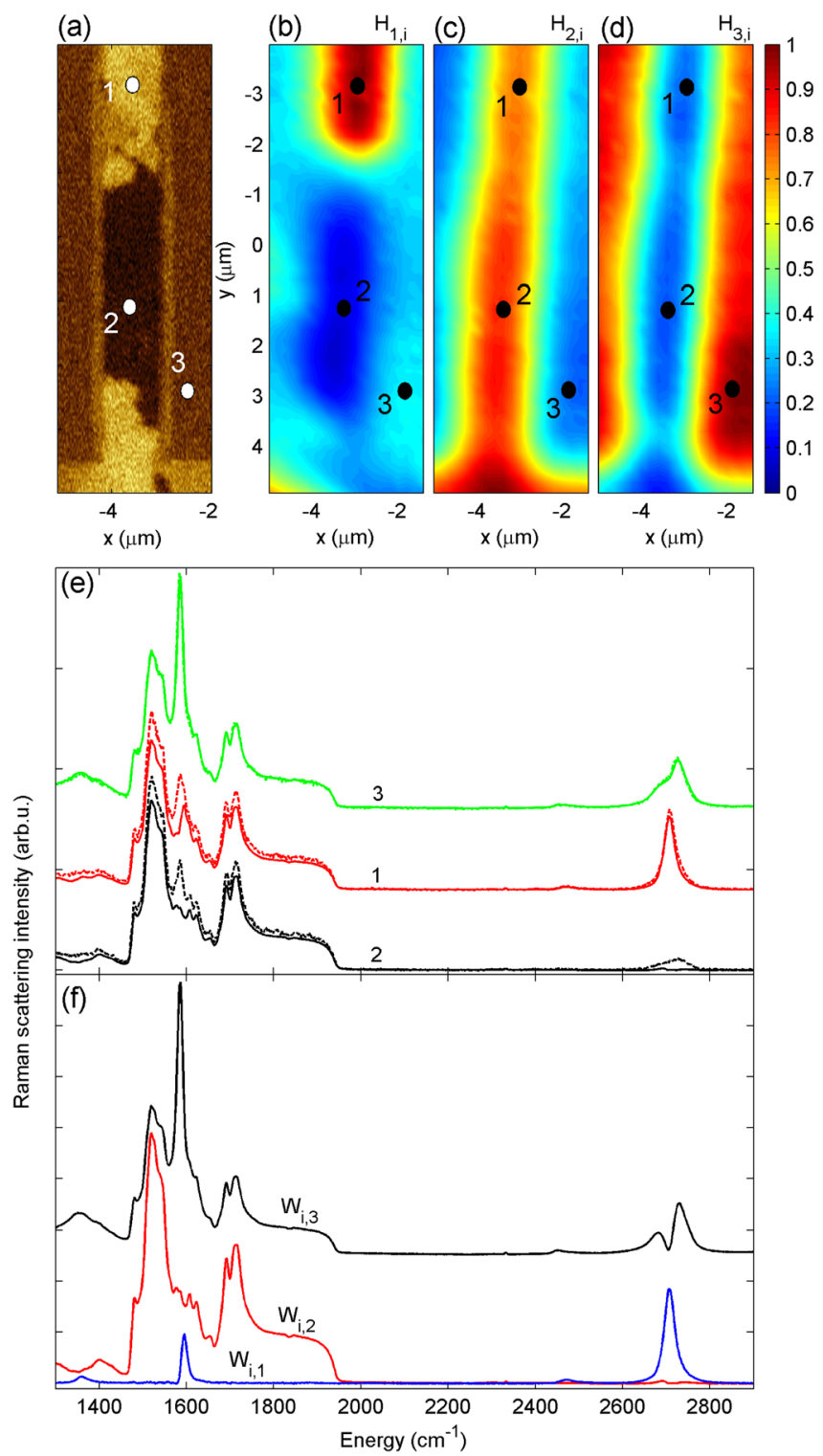

FIG. 2. (a) Electrostatic force microscopy image. Maps of the linear coefficients of (b) single layer graphene, (c) $\mathrm{SiC}$ and (d) multilayer graphene of the same area on the sample. (e) Normalized data (dashed lines) measured at points 1, 2, and 3 (shown in (a)-(d) by white and black numbered circles) are compared to NMF filtered spectra (solid lines) using threshold 0.3. (f) Blue, red and black spectra are basis functions corresponding to linear coefficients in (b), (c), and (d), respectively. can be due to the contribution of $\mathrm{AB}$ stacked layers (about $10 \%-15 \%$ of the stacking ${ }^{29}$ ) or small graphitic areas. ${ }^{12}$

Because we clearly identified three regions, the Raman spectra at each point of the mapping were decomposed by NMF of rank $k=3$. The three basis functions $W_{i, 1}, W_{i, 2}, W_{i, 3}$ (columns of matrix $W$ ) are plotted in Fig. 2(f). The $W_{i, 1}$ and $W_{i, 2}$ basis function can clearly be associated with single layer graphene and bare $\mathrm{SiC}$, respectively, whereas $W_{i, 3}$ is more complex. The linear coefficients $H_{1, i}, H_{2, i}$, and $H_{3, i}$ (rows of matrix $H$ ) plotted in Figs. 2(b)-2(d) show that each basis function $W_{i, 1}, W_{i, 2}$, and $W_{i, 3}$ clearly dominates in a different area of the sample. Particularly, area 2 shows well pronounced minimum for both graphene related basis functions $W_{i, 1}$ and $W_{i, 3}$.

The channel between the MEG pads ( $0.9 \mu \mathrm{m}$ wide $)$ is narrower than the laser beam spot $(1.2 \mu \mathrm{m}$, as determined above); therefore, the Raman signal from the channel (areas 1 and 2) gets contribution also from the MEG pads. The Raman spectra of just the narrow channel can nevertheless be reconstructed, by keeping only the largest contributions to the Raman signal in each area. For this, we performed an inverse data composition $V=W H$ by replacing the linear coefficients in matrix $H$ by $H^{\prime}$, where $H_{i j}^{\prime}=H_{i j}$ for $H_{i j}>H_{t h}=0.3$ otherwise $H_{i j}^{\prime}=0$. Applying this threshold on the inverse data composition gives the filtered Raman spectra of Fig. 2(e) (solid lines). The graphene related signal in area 1 is still present; however, the $\mathrm{D}, \mathrm{G}$, and 2D peaks are filtered out at point 2. Changing the threshold $H_{t h}$ sets the sensitivity of the filtering. At low (high) value of $H_{t h}$ the measured data (basis functions), respectively, are retrieved. The filtered Raman spectra prove that area 1 is a single layer as shown by its weak G-peak and narrow Lorentzian 2D peak $\left(F W H M=27 \mathrm{~cm}^{-1}\right)$. Area 3 is covered by few layer graphene (strong G-peak, splitted 2D peak, that may come from layers of different strain or doping in the MEG stack $^{11,30}$ and attenuated intensity of $\mathrm{SiC}$ Raman scattering ${ }^{16}$ ), as expected from the growth conditions. Area 2 is bare $\mathrm{SiC}$, showing that the regraphitization process in the trench is only partial.

We have shown here that using a threshold $H_{t h}$ in signal re-composition effectively removes non-local spurious signal and plays a similar role as deconvolution. It is important to note that, contrary to deconvolution, this method does not require knowledge of the impulse response function.

We note however that the NMF spectral decomposition is not unique. Each decomposition $V=W H$ can be replaced by $V=\tilde{W} \tilde{H}=W D^{-1} D H$, for any non-negative regular square matrix $D$. The non-uniqueness can be dealt with by adding constraints on the $W$ or $H$ matrices. The most common constraints are sparsness, ${ }^{20,24}$ regularity, ${ }^{20}$ orthogonality, ${ }^{31}$ and constraint on the minimum amplitude of the recovered spectra. ${ }^{32}$ The orthogonality constraint has been discussed $^{32}$ to be often inconvenient due to possibility of highly correlated signals composing the experimental data. The constraint on the minimum amplitude of the recovered spectra deals with the negative experimental data caused by noise, however, since the noise level in our data was low enough, we have not applied this method here. The ambiguity of NMF is also reflected in our analysis. For instance, the basis function $W_{i, 3}$ (Fig. 2(f)) is a mixture of graphene and SiC Raman signals. The mixed basis function $W_{i, 3}$ can be 


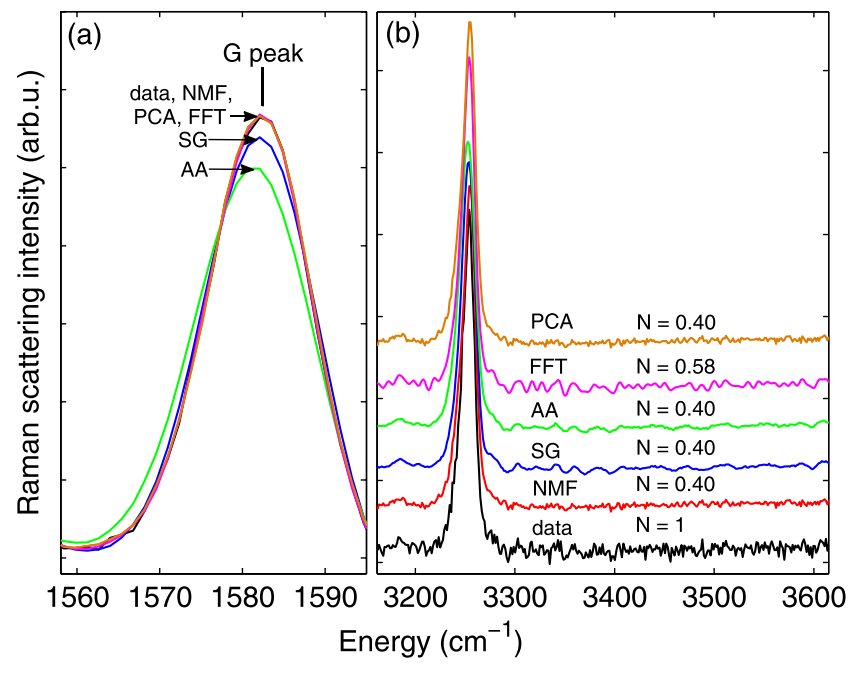

FIG. 3. Raman spectra of epitaxially grown graphene on SiC. NMF is used for data (black curve, marked as data) smoothing instead of decomposition. Several smoothing algorithms are compared. Red, blue, green, magenta, and yellow curves show smoothed data using 2 component NMF, 2nd order Savitzky-Golay with winnow length 15 points (SG), adjacent averaging using 8 point rectangular smoothing window (AA), high-pass Fourier transform filter (FFT, high-pass frequency cut off $0.12 \mathrm{~cm}$ ) and 2 component PCA algorithms. Influence of data smoothing on the (a) G-peak intensity and (b) flat part of the spectrum is shown.

further decomposed either by applying different NMF constraints, by finding a transformation matrix $D$ or by a Raman depth analysis, as discussed above (Fig. 1).

Next, we use NMF for data smoothing. As explained above, the exact NMF data decomposition is $V_{\text {exp }}=W H+E$, where the Frobenius norm of matrix $E$ is minimized by the NMF algorithm. Therefore, matrix $E$ contains mainly the noise and the product $V=W H$ carries the smoothed experimental data. We use the Raman spectra measured on the first MEG sample to compare NMF smoothing with four most frequently used smoothing algorithms (see Fig. 3). We define the normalized data noise level by the ratio $N=N_{s m} / N_{\text {exp }}$ of the standard deviation of the smoothed data $N_{s m}$ to the standard deviation of the experimental data $N_{\text {exp }}$. The smoothing parameters of Savitzky-Golay (SG), adjacent averaging (AA), and Fast Fourier Transform high pass filter (FFT) are set so that the data noise level is reduced to the level of NMF 2component smoothing $N=0.4$. We compare the noise level in a region of the spectrum with no peak in Fig. 3(b) and the intensity of the sharp G-peak (that sits on top of the SiC peaks) in Fig. 3(a). SG and AA smoothing already influence the intensity of G-peak and high pass FFT filter does not reduce the noise level below $N=0.58$. Worse, slowly varying regions of the Raman spectrum are modified also when the low-pass FFT filter is used. PCA and NMF smoothing both reduce the noise level to $N=0.4$ and keep the sharp G-peak intensity. Hence, both NMF and PCA provide the most efficient noise reduction without modifying any information contained in a Raman spectrum of graphene epitaxially grown on $\mathrm{SiC}$.

The non-negative matrix factorization method was applied to decompose the Raman scattering spectra of epitaxial graphene into pure graphene and $\mathrm{SiC}$ spectra. In contrast to simple commonly used $\mathrm{SiC}$ background subtraction, the method provides well defined spectra, with much reduced noise and non-negative intensity even for monolayer graphene. The method provides also the relative contribution of each spectrum, which is used for spatial mapping of patterned samples and depth profile. In particular, pure Raman spectrum of sub micron size graphene structures can be extracted. Finally, we have demonstrated that NMF is efficient for data smoothing and deconvolution, with no assumption required for the functional form of the signals, and in combination with two-dimensional nature of graphene layers, it can be used as a method to characterize the laser beam profiles.

We acknowledge financial support from NSF-MRSEC (\#DMR-0820382), AFSOR, the W. M. Keck foundation, and the Partner University Fund. We also thank the reviewer for pointing out two references. ${ }^{31,32}$

${ }^{1}$ C. Berger, Z. Song, T. Li, X. Li, A. Y. Ogbazghi, R. Feng, Z. Dai, A. N. Marchenkov, E. H. Conrad, P. N. First, and W. A. de Heer, J. Phys. Chem. B 108, 19912-19916 (2004).

${ }^{2}$ M. Sprinkle, M. Ruan, Y. Hu, J. Hankinson, M. Rubio-Roy, B. Zhang, X. Wu, C. Berger, and W. A. de Heer, Nat. Nanotechnol. 5, 727-731 (2010).

${ }^{3}$ Y.-M. Lin, A. Valdes-Garcia, S.-J. Han, D. B. Farmer, I. Meric, Y. Sun, Y. Wu, C. Dimitrakopoulos, A. Grill, P. Avouris, and K. A. Jenkins, Science 332, 1294-1297 (2011).

${ }^{4}$ Z. Guo, R. Dong, P. S. Chakraborty, N. Lourenco, J. Palmer, Y. Hu, M. Ruan, J. Hankinson, J. Kunc, J. D. Cressler, C. Berger, and W. A. de Heer, Nano Lett. 13, 942 (2013).

${ }^{5}$ J. S. Moon, H.-C. Seo, M. Antcliffe, D. Le, C. McGuire, A. Schmitz, L. O. Nyakiti, D. K. Gaskill, P. M. Campbell, K.-M. Lee, and P. Asbeck, IEEE Electron. Device Lett. 34, 465-467 (2013).

${ }^{6}$ B. Dlubak, M.-B. Martin, C. Deranlot, B. Servet, S. Xavier, R. Mattana, M. Sprinkle, C. Berger, W. A. De Heer, F. Petroff, A. Anane, P. Seneor, and A. Fert, Nat. Phys. 8, 557-561 (2012).

${ }^{7}$ M. Ruan, Y. Hu, Z. Guo, R. Dong, J. Palmer, J. Hankinson, C. Berger, and W. A. de Heer, MRS Bull. 37, 1138-1147 (2012).

${ }^{8}$ J. Baringhaus, C. Tegenkamp, F. Edler, M. Ruan, E. Conrad, C. Berger, and W. A. de Heer, e-print arXiv:1301.5354 [cond-mat.mes-hall].

${ }^{9}$ J. Hicks, A. Tejeda, A. Taleb-Ibrahimi, M. S. Nevius, F. Wang, K. Shepperd, J. Palmer, F. Bertran, P. L. Fvre, J. Kunc, W. A. de Heer, C. Berger, and E. H. Conrad, Nature 9, 49-54 (2013).

${ }^{10}$ A. C. Ferrari, J. C. Meyer, V. Scardaci, C. Casiraghi, M. Lazzeri, F. Mauri, S. Piscanec, D. Jiang, K. S. Novoselov, S. Roth, and A. K. Geim, Phys. Rev. Lett. 97, 187401 (2006).

${ }^{11}$ L. Malard, M. Pimenta, G. Dresselhaus, and M. Dresselhaus, Phys. Rep. 473, 51-87 (2009).

${ }^{12}$ C. Faugeras, A. Nerriere, M. Potemski, A. Mahmood, E. Dujardin, C. Berger, and W. de Heer, Appl. Phys. Lett. 92, 011914 (2008).

${ }^{13}$ D. Lee, C. Riedl, B. Krauss, K. von Klitzing, U. Starke, and J. H. Smet, Nano Lett. 8, 4320 (2008).

${ }^{14}$ Z. H. Ni, W. Chen, X. F. Fan, J. L. Kuo, T. Yu, A. T. S. Wee, and Z. X. Shen, Phys. Rev. B 77, 115416 (2008).

${ }^{15}$ J. Rohrl, M. Hundhausen, K. V. Emtsev, T. Seyller, R. Graupner, and L. Ley, Appl. Phys. Lett. 92, 201918 (2008).

${ }^{16}$ S. Shivaraman, M. Chandrashekhar, J. Boeckl, and M. Spencer, J. Electron. Mater. 38, 725-730 (2009).

${ }^{17}$ W. A. de Heer, C. Berger, X. Wu, Y. Hu, M. Ruan, J. Stroscio, P. First, R. Haddon, B. Piot, C. Faugeras, M. Potemski, and J. S. Moon, J. Phys. D 43, 374007 (2010).

${ }^{18}$ M. Suemitsu and H. Fukidome, J. Phys. D 43, 374012 (2010).

${ }^{19}$ D. D. Lee and H. S. Seung, Nature 401, 788-791 (1999).

${ }^{20} \mathrm{~J}$. Kim and H. Park, in Proceedings of the 2008 Eighth IEEE International Conference on Data Mining (ICDM'08), pp. 353-362 (2008), code available at http://www.cc.gatech.edu/ hpark/nmfsoftware.php.

${ }^{21}$ H. Kim and H. Park, SIAM J. Matrix Anal. Appl. 30, 713-730 (2008).

${ }^{22}$ W. A. de Heer, C. Berger, M. Ruan, M. Sprinkle, X. Li, Y. Hu, B. Zhang, J. Hankinson, and E. Conrad, Proc. Natl. Acad. Sci. U.S.A. 108, 16900-16905 (2011).

${ }^{23}$ D. A. Schmidt, T. Ohta, and T. E. Beechem, Phys. Rev. B 84, 235422 (2011). 
${ }^{24}$ P. O. Hoyer, J. Mach. Learn. Res. 5, 1457-1469 (2004).

${ }^{25}$ W. Windl, K. Karch, P. Pavone, O. Schütt, D. Strauch, W. H. Weber, K. C. Hass, and L. Rimai, Phys. Rev. B 49, 8764-8767 (1994).

${ }^{26}$ J. C. Burton, L. Sun, F. H. Long, Z. C. Feng, and I. T. Ferguson, Phys. Rev. B 59, 7282-7284 (1999).

${ }^{27}$ B. Krauss, T. Lohmann, D.-H. Chae, M. Haluska, K. von Klitzing, and J. H. Smet, Phys. Rev. B 79, 165428 (2009).

${ }^{28}$ B. E. A. Saleh and M. C. Teich, Fundamentals of Photonics (John Wiley \& Sons, Inc., Hoboken, New Jersey, 2007).
${ }^{29}$ J. Hicks, K. Shepperd, F. Wang, and E. H. Conrad, J. Appl. Phys. D 45, 154002 (2012).

${ }^{30}$ L. M. Malard, J. Nilsson, D. C. Elias, J. C. Brant, F. Plentz, E. S. Alves, A. H. Castro Neto, and M. A. Pimenta, Phys. Rev. B 76, 201401 (2007).

${ }^{31}$ H. L. Li, T. L. Adali, and W. Wang, J. VLSI Signal Proc. Syst. Signal, Image, and Video Technol. 48, 83-97 (2007); and IEEE Workshop on Machine Learning for Signal Processing (MLSP), Mystic, CT September 28-30 (2005).

${ }^{32}$ P. Sajda, S. Du, and L. Parra, Proc. SPIE 5207, 321-331 (2003). 